\title{
A Case Report and Review of the Literature on Uterine Fatty Tumours (UFT): A Field of Heterogeneous Data
}

\author{
Luca Bernardini ${ }^{\star *}$, A Zacutti ${ }^{1}$, N Gorji ${ }^{2}$, ML Giannoni ${ }^{1}$, Francesca Accorsi ${ }^{1}$ and E Volpi ${ }^{1}$ \\ ${ }^{1}$ Department of Obstetrics and Gynecology, Saint'Andrew Hospital, ASL 5, Via Vittorio Veneto 197, 19100, La Spezia, Italy \\ ${ }^{2}$ S.C. Anatomia ed Istologia Patologica, Saint'Andrew Hospital, ASL 5, Via Vittorio Veneto 197, 19100, La Spezia, Italy
}

\begin{abstract}
A case of uterine fatty tumour of $2 \mathrm{~cm}$ studied by transvaginal ultrasound in a 67-year-old woman undergone totally laparoscopic hysterectomy is here presented along with an updated review of this kind of tumours. A total of 226 cases from 75 publications could be found. Traditional definitions of these tumours are here critically reconsidered. The terminology used to define these tumours is confusing and reflects main radiological and pathological points of view (uterine fatty tumours [UFT] vs. lipoma/ lipoleiomyoma). It is here stressed that these tumours by occurring in aged and overweight women are not so uncommon as generally believed, can be very well recognized, as far as they are small $(<4 \mathrm{~cm})$, by transvaginal ultrasound while CT and MR become more specific for decision making in case of larger size tumours. Symptoms largely vary from none to sudden lump enlargement or bleeding or pain. An association with malignancy has been found in $10 \%$ suggesting a radical treatment (hysterectomy) in most cases.
\end{abstract}

\section{Case Report}

Since first description by Lobstein in 1816 until 1966 only 131 cases of lipomatous uterine tumours (UFT) were initially reported. From the 70's up to date this number has been progressively growing to at least 357 cases around the world. The first preoperative study on a case of uterine "fatty" tumour has been described by Jacobs and Markowitz in 1988 [1] and since then the preoperative imaging study of these tumours has significantly increased due to the expansion of the radiological technologies and diffusion of ultrasonography in routine gynaecologic activity. Uterine fatty tumours (UFT) or lipoleiomyomas (LLM) are a kind of leiomyomas with prevalent fatty component occurring mostly in peri-menopausal and postmenopausal obese women. Many clinical and pathological aspects of these uncommon uterine nodules have been already reported [2-6]. Nonetheless literature largely varies with a prevalence of radiological case-reports for clinical data and histo-pathological review analysis on pathogenesis investigation. Little or nothing about UFT is found in important gynaecological journals. Moreover it is not clear which diagnostic tool among ultrasound, CT and MR should be best utilized for clinical follow up of these nodules $[7,8]$, whether hysterectomy should or not always warranted $[9,10]$ and how frequently a malignant transformation of these tumours should also be expected $[3,11]$. We report here a single case of UFT along with a full literature review on this topic with the aim to help gynaecologists in the clinical counselling of these tumours.

A 67 year old woman with no clinical symptoms or relevant medical history (menopause at 54 y.o., hypercholesterolemia and overweight [BMI=28.9]) was found with a $1.9 \mathrm{~cm}$ hyper echoic uterine nodule on the intramural/subserosal margin of anterior wall. This little hyper echoic nodule was clearly distinguished from the surrounding myometrium without showing any posterior acoustic shadow. Contrary to that generally reported this lesion appeared not to be encased in hypo echoic ring (Figure 1). The discovery of this nodule was made occasionally during routine transvaginal ultrasound (US). Following CT and MR (images here not reproduced) a presumptive diagnosis of uterine fatty tumour was made and patient advised to undergo surgery. A totally laparoscopic hysterectomy with bilateral adnexectomy was performed and successively the resulted histopathological analysis confirmed the benign nature of the lesion. Pathological findings: grossly, uterus weight of 76 grams and

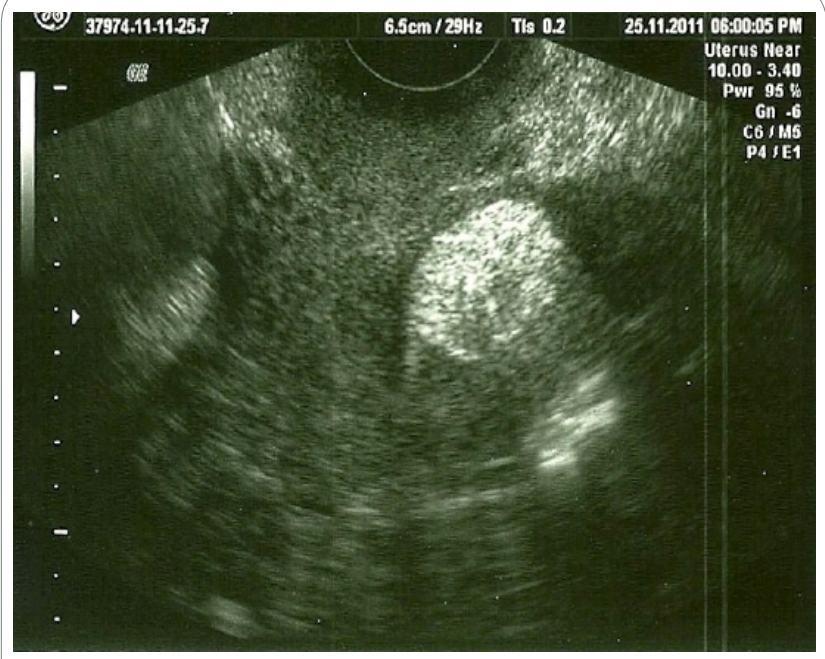

Figure 1: TSV ultrasound showing a typical hyperechogenic intramural nodule of $2 \mathrm{~cm}$.

dimensions of $7 \times 4 \times 3 \mathrm{~cm}$, at the cut surface with a yellowish intramural nodule of corpus uteri of $1.9 \mathrm{~cm}$ in diameter, with welldefined edges but non encapsulated; the overlying endometrium was atrophic and the cervix showed no significant macroscopic changes (Figure 2). Microscopically the tumour was predominantly made up of mature adipose tissue mingled with bundles of smooth muscle (positive for smooth muscle actin antibody at immunohistochemistry). The diagnosis of lipoleiomyoma was made. The patient gave her informed consent to the study.

"Corresponding Author: Dr. Luca Bernardini, Department of Obstetrics and Gynecology, Saint'Andrew Hospital, ASL 5, Via Vittorio Veneto 197, 19100, La Spezia, Italy; E-mail: ostgin-sarzana@libero.it

Citation: Bernardini L, Zacutti A, Gorji N, Giannoni ML, Accorsi F, et al. (2014) A Case Report and Review of the Literature on Uterine Fatty Tumours (UFT): A Field of Heterogeneous Data. Int J Gynecol Clin Pract 1: 101. doi: http://dx.doi. org/10.15344/2394-4986/2014/101

Copyright: (c) 2014 Bernardini et al. This is an open-access article distributed under the terms of the Creative Commons Attribution License, which permits unrestricted use, distribution, and reproduction in any medium, provided the original author and source are credited. 


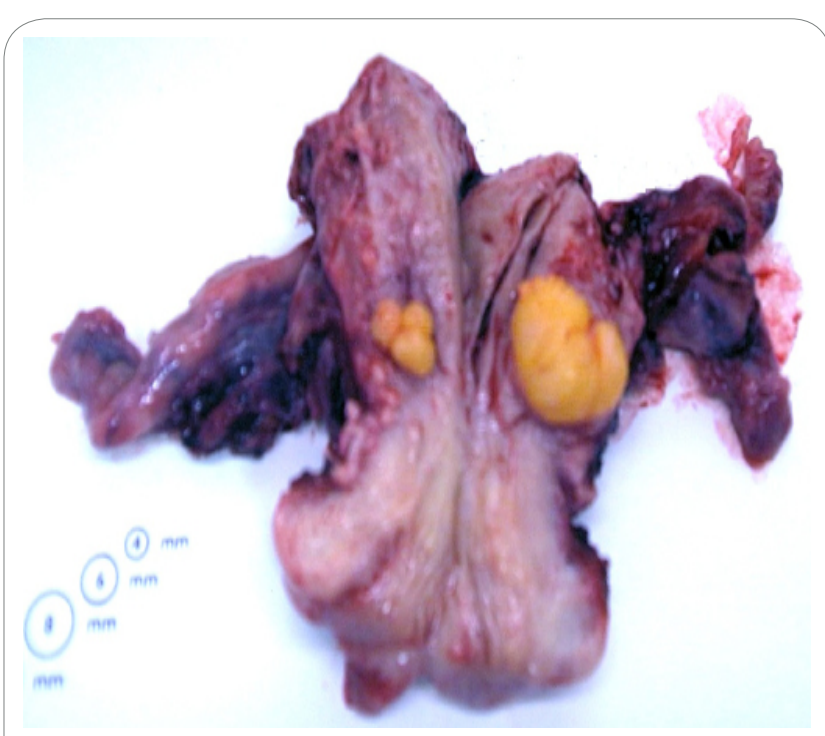

Figure 2: Macroscopic pathology of fatty tumour.

Overall a total of 226 fatty tumours of the uterus including lipomas, lipoleiomyomas, liposarcomas and endometrial cancers associated with lipoleiomyomas could be retrieved from the literature. We started reviewing data from the study of Willen et al 1978 [2] (the first 131 cases of UFT from 1816 to 1966 were excluded). This has corresponded to 75 independent studies here separately reported in 3 different tables: Table 1 for all clinical studies $(n=46)$, Table 2 for pathological review data analysis $(n=16)$ and Table 3 for malignant uterine tumours with fatty component and endometrial cancers coincidental with benign fatty tumours $(n=13)$. Mean patient age, tumour size and incidence of UFT (which varied accordingly to the criteria used being low - $0.03 \%$ - in hysterectomy and higher - $0.8-2.1 \%$ in uterine leiomyomas studies) along with symptoms and methodology used for preoperative tumour study and detection are summarized in table 4. No association whatsoever between aging and tumour size has been noted (Table 1). Despite being a tumour associated with aging we observed a prevalence of $23 \%$ in women younger than 54 years. As for all the other uterine fibroids, clinical signs largely varied including occasional discovery in asymptomatic patients, bleeding, abdominal or pelvic pain, pelvic mass discovery, liver problems, anaemia, disuria, and constipation. Despite UFT are said to recur more frequently in overweight and obese perimenopausal women, specific details on body weight or BMI have in general not been reported. Diagnosis and radiological follow up has been obtained in different ways including a variable utilization of multiple technologies such as US, CT, MR. All 3 of these have been employed only in 16/46 independent case reports. US either by transvaginal (TSV-US) or abdominal (TA-US) route was used in 42/46 studies but TVS has been applied only in 19 occasions. The use of TVS alone as unique diagnostic technique has been used only in 4 studies (for a total of 10 patients) while MR alone, as unique diagnostic tool, in 2 studies (for a total of 10 patients). Hysterectomy has been generally the rule for most patients but 9 (7 study reports). By a pathologist point of view, most of the uterine fatty tumours were lipoleiomyomas $(n=135)$, less frequently occurred lipomas $(n=18)$, angiomyolipomas $(n=4)$, and others (1 atypical lipoleiomyoma, 1 bizarre epitheliod lipoleiomyoma and 1 plexiform lipoleiomyoma, 1 giant lipoleiomyoma, 1 myolipoma of round ligament). An intramural or subserosal location has been found in most cases despite the possibility to encounter everywhere around the uterus these tumours (round ligament, cervix, or as pelvic mass). Satellite fibroid tumours or leiomyomas were described in $33 \%$ of the cases. Notably, in $4 / 18$ lipomas a concomitant presence of an endometrial cancer was discovered. To the date, liposarcomas and lipoleiomyosarcomas have been found to be described in at least 8 independent studies for a total of 19 patients.

\section{Discussion}

In the last months the number of publications on UFT has grown a lot with increasing online diffusion of radiological images [12-14]. In these imaging reports, lipoleiomyomas are defined as uncommon, benign tumours not requiring surgical treatment. Most data on these tumors have been published on radiology medicine journals or reviews of pathology archives. Little is given on gynaecological journals. This is of matter since for gynaecologists it would be of value a prompt recognition and counselling of these tumours when performing ultrasound. This is truer in case of overweight peri-menopausal women who have fibroids in almost $80 \%$ of the cases [15]. Since the incidence of UFT in older patients is higher than $1 \%$ it is questionable to consider UFT as uncommon tumours as yet. Particularly, when the aging trend of the world population is considered. As far as it refers to clinical management a dramatic variability is from one study to another. This is a consequence of the variability of the tumour size, presence or not of symptoms and interpretation of imaging investigation results. As originally reported by Pham et al [16] and others $[17,18]$ when these fatty tumours are small $(2-5 \mathrm{~cm})$ and of certain uterine origin, transvaginal ultrasound is very sensitive and there is no need of additional and more specific technologies (CT and MR). Since correct diagnosis can only be expressed after histologic examination and malignancy be found, we believe that in general hysterectomy should be always done. Myomectomy could be an option only for younger patients scheduled to special infertility cures (i.e. oocyte donation cycles). The review of literature data shows that the percentage of UFT occurring in women $<54$ years is not insignificant (23\%) (3). A conservative management is mandatory in conditions contraindicating surgery when surveillance by means of ultrasonography, CT, and MR is rather coupled to uterine artery embolization $[19,15]$. When clinical manifestations such as sudden lump enlargement or pelvic masses are present, the implementation with CT and MR becomes mandatory. Despite the very high specificity of MR for detecting origin and mass constitution (fat tissue), the diagnosis is made only after excluding other pelvic masses (benign cystic ovarian teratoma, malignant degeneration of cystic teratoma, lipomatous ovarian tumour, pelvic lipoma, liposarcoma and lipoblastic lymphadenopathy) $[9,10,20,21]$. CT and MR have allowed valid follow up of pelvic masses in one patient with severe medical contraindication to surgery [7] but pitfalls in imaging interpretation may always happen and one case with fatal consequences due to unnecessary surgery (lipoleiomyoma misdiagnosed as liposarcoma - patient died post hysterectomy) has been reported [22]. Although most fibroids regress after the menopause the UFT are more frequent in elderly women. It has been estimated that the prevalence of uterine lipoleiomyoma in patients older than 80 years is close to $10 \%$ (5/50 uterine lipoleiomyomas) [3] and it is well known that elderly women have a higher risk of perioperative morbidity and mortality. Therefore sometime correct counselling of these lesions is not easy in particular considering the finding of an association with sarcomas and endometrial cancers in $10 \%$ of the cases. Literature description of the lipomatous uterine tumours is highly variable as much as it is the biology of these tumours, the histotype and also the criteria used 
Citation: Bernardini L, Zacutti A, Gorji N, Giannoni ML, Accorsi F, et al. (2014) A Case Report and Review of the Literature on Uterine Fatty Tumours (UFT): A Field of Heterogeneous Data. Int J Gynecol Clin Pract 1: 101. doi: http://dx.doi.org/10.15344/2394-4986/2014/101

Page 3 of 7

\begin{tabular}{|c|c|c|c|c|c|c|c|c|c|c|}
\hline $\begin{array}{l}\text { No. of } \\
\text { cases }\end{array}$ & Size & Age & Symptoms & $\begin{array}{l}\text { US } \\
(\mathrm{TA}+\mathrm{TSV})\end{array}$ & $\mathrm{CT}$ & MR & Surg. & Histology & Fibroids & References \\
\hline 2 & $7 \mathrm{~cm}$ & & $\begin{array}{l}\text { Abdominal pain } \\
\text { lump growth }\end{array}$ & TA & yes & yes & yes & Lipoma & no & $\begin{array}{l}\text { Bachor, Baczako. Geburtshife Frauenheilkd } \\
\text { 46;842-3, } 1986\end{array}$ \\
\hline 1 & $\begin{array}{l}2 \mathrm{~cm} \\
5 \mathrm{~cm}\end{array}$ & & Asymptomatic & $\mathrm{TA}$ & yes & no & yes & Lipoma & no & $\begin{array}{l}\text { Jacobs and Markowitz. AJR:150;1335-1336, } \\
1988\end{array}$ \\
\hline 2 & & & & $\begin{array}{l}\text { TA } \\
\text { TA }\end{array}$ & $\begin{array}{l}\text { yes } \\
\text { yes }\end{array}$ & $\begin{array}{l}\text { yes } \\
\text { yes }\end{array}$ & $\begin{array}{l}\text { yes } \\
\text { yes }\end{array}$ & $\begin{array}{l}\text { Lipoma } \\
\text { Lipoleiomyoma }\end{array}$ & $\begin{array}{l}\text { no } \\
\text { no }\end{array}$ & Dodd III and Budzik. AJR:155;317-322,1990 \\
\hline 1 & & & & & yes & yes & yes & Lipoleiomyoma & & $\begin{array}{l}\text { Aizenstein et al. Gynecol Oncol:40;274-6, } \\
1991\end{array}$ \\
\hline 1 & & & & TA & no & no & yes & Lipoleiomyoma & no & $\begin{array}{l}\text { Ekici, Vicdan. Int J Gynaecol } \\
\text { Obstet:42;167-71, } 1993\end{array}$ \\
\hline 1 & $9 \mathrm{~cm}$ & 59 & Abnormal liver enzymes & TA & yes & yes & yes & Lipoleiomyoma & yes & $\begin{array}{l}\text { Villanueva et al. Abdom Imaging:18;402-3, } \\
1993\end{array}$ \\
\hline 1 & & 43 & & TA & no & no & yes & Lipoleiomyoma & no & Pham et al. Can Assoc Radiol:44;463-5,1993 \\
\hline 1 & $20 \mathrm{~cm}$ & 44 & Asymptomatic & & & yes & yes & $\begin{array}{l}\text { Myolipoma of the } \\
\text { round ligament }\end{array}$ & no & $\begin{array}{l}\text { Sonobe et al. Virchows Arch:427;455-458, } \\
1995\end{array}$ \\
\hline 1 & & & & $\mathrm{TA}$ & yes & yes & yes & Lipoma & no & $\begin{array}{l}\text { Alonso et al. Ginecol Obstet Mex:63;30-2, } \\
1995\end{array}$ \\
\hline 11 & & & & $\begin{array}{l}\text { TA }(n=5) \\
\text { TSV }(n=6)\end{array}$ & no & no & yes & Lipoleiomyoma & $2 / 11$ & $\begin{array}{l}\text { Serafini et al. J Ultrasound Med:15;195-99, } \\
1996\end{array}$ \\
\hline 1 & $8 \mathrm{~cm}$ & 73 & Pelvic Mass & TA & yes & yes & yes & Lipoleiomyoma & no & $\begin{array}{l}\text { Tsushima et al. British J Rad:70;1068-1070, } \\
1997\end{array}$ \\
\hline 1 & & 62 & Cholelithiasis & & yes & yes & yes & Lipoleiomyoma & no & $\begin{array}{l}\text { Ishigami et al. Abdom Imaging:23;214-216, } \\
1998\end{array}$ \\
\hline 2 & $\begin{array}{l}6.8 \mathrm{~cm} \\
5.5 \mathrm{~cm}\end{array}$ & $\begin{array}{l}55 \\
62\end{array}$ & $\begin{array}{l}\text { Asymptomatic } \\
\text { Bleeding }\end{array}$ & $\begin{array}{l}\text { TA } \\
\text { TA }\end{array}$ & $\begin{array}{l}\text { yes } \\
\text { yes }\end{array}$ & & $\begin{array}{l}\text { yes } \\
\text { yes }\end{array}$ & $\begin{array}{l}\text { Lipoleiomyoma } \\
\text { Lipoleiomyoma }\end{array}$ & $\begin{array}{l}\text { yes } \\
\text { yes }\end{array}$ & $\begin{array}{l}\text { Prieto et al. Abdom Imaging:25;655-657, } \\
2000\end{array}$ \\
\hline 1 & & & Large tumor & & yes & & yes & Lipoleiomyoma & no & $\begin{array}{l}\text { Su et al. Eur J Gynaecol Oncol:22;439-40, } \\
2001\end{array}$ \\
\hline 1 & $5 \mathrm{~cm}$ & 48 & Pelvic pain & TA & yes & yes & Biopsy & Lipoleiomyoma & no & Avritsher et al. AJR:177;856-857, 2001 \\
\hline 1 & & 51 & Bleeding & TSV & no & no & yes & Angiolipoma & no & Braun et al, 2002 \\
\hline 1 & $5.5 \mathrm{~cm}$ & 67 & $\begin{array}{l}\text { Low Haematocrit } \\
\text { Warfarin therapy }\end{array}$ & TSV & yes & & no & $\mathrm{UFT}^{\circ}$ & yes & Chan et al. JHK Coll Radiol:6;30-32, 2003 \\
\hline 1 & & 67 & Abdominal pain & & yes & yes & yes & Lipoma & no & $\begin{array}{l}\text { Al-Maghrabi et al. Saudi Med J:25;1492-4, } \\
2004\end{array}$ \\
\hline 1 & $10 \mathrm{~cm}$ & 65 & Pelvic mass & TA & yes & yes & yes & Lipoleiomyoma & no & $\begin{array}{l}\text { Chawla et al. Applied Radiology Online } \\
\text { 38-40, April } 2004\end{array}$ \\
\hline 1 & $7.8 \mathrm{~cm}$ & 62 & Bleeding & TA & yes & no & yes & Lipoma & no & Lau and Thoeni. British J Rad:78;72-74, 2005 \\
\hline 1 & $7 \mathrm{~cm}$ & 75 & Abdominal pelvic pain & TSV & yes & yes & yes & Lipoma & yes & $\begin{array}{l}\text { Coumbaras et al. Abdom } \\
\text { Imaging:30;239-241, } 2005\end{array}$ \\
\hline 1 & & & & TA & & & & Lipoma & & $\begin{array}{l}\text { Harish et al. Indian J Pathol } \\
\text { Microbiol:48;377-8,2005 }\end{array}$ \\
\hline 1 & $5 \mathrm{~cm}$ & 53 & Bleeding and pain & TSV & no & no & yes & Lipoleiomyoma & yes & Alper et al. Malta Medical J:17;40-41, 2005 \\
\hline 1 & $5 \mathrm{~cm}$ & 65 & $\begin{array}{l}\text { Bleeding and abdominal } \\
\text { lump growth }\end{array}$ & TSV & no & no & yes & Lipoma & no & Deb et al. MJAFI:61;385-386, 2005 \\
\hline 1 & $12 \mathrm{~cm}$ & 72 & Pelvic mass & TA & yes & no & yes & Lipoleiomyoma & no & Arikawa et al. Kurume Med J:53;37-40, 2006 \\
\hline 1 & $17 \mathrm{~cm}$ & 52 & Pelvic mass & TSV & yes & yes & yes & Lipoma & no & $\begin{array}{l}\text { Fujimoto et al. J Obstet } \\
\text { Gynaecol:32;520-523,2006 }\end{array}$ \\
\hline 1 & $12 \mathrm{~cm}$ & 60 & Pelvic mass & TSV & yes & yes & yes & Lipoma & yes & $\begin{array}{l}\text { Fernandes et al. Indian J Pathol } \\
\text { Microbiol:50;800-1, } 2007\end{array}$ \\
\hline
\end{tabular}

Table 1 continued.. 
Citation: Bernardini L, Zacutti A, Gorji N, Giannoni ML, Accorsi F, et al. (2014) A Case Report and Review of the Literature on Uterine Fatty Tumours (UFT): A Field of Heterogeneous Data. Int J Gynecol Clin Pract 1: 101. doi: http://dx.doi.org/10.15344/2394-4986/2014/101

Page 4 of 7

\begin{tabular}{|c|c|c|c|c|c|c|c|c|c|c|}
\hline 9 & $\begin{array}{l}9 \mathrm{~cm} \\
10 \mathrm{~cm} \\
10 \mathrm{~cm} \\
9 \mathrm{~cm} \\
4 \mathrm{~cm} \\
3.5 \mathrm{~cm} \\
6 \mathrm{~cm} \\
6.5 \mathrm{~cm} \\
4.5 \mathrm{~cm}\end{array}$ & $\begin{array}{l}65 \\
76 \\
64 \\
47 \\
55 \\
49 \\
64 \\
70 \\
55\end{array}$ & $\begin{array}{l}\text { Abdominal pain } \\
\text { Abdominal pain } \\
\text { Abdominal pain } \\
\text { Abdominal pain } \\
\text { Bleeding } \\
\text { Bleeding } \\
\text { Bleeding } \\
\text { Dysuria } \\
\text { Anaemia }\end{array}$ & no & no & yes & yes & Lipoleiomyoma & $6 / 9$ yes & $\begin{array}{l}\text { Kitajima et al. AJR:189;W100-104, } \\
2007\end{array}$ \\
\hline 1 & & & & $\mathrm{TA}$ & yes & yes & no & $\mathrm{UFT}^{\circ}$ & no & $\begin{array}{l}\text { Erdem et al. Magn Reson } \\
\text { Imaging:25;1232-6, } 2007\end{array}$ \\
\hline 1 & $11 \mathrm{~cm}$ & 60 & Cholecystitis & $\mathrm{TA}$ & yes & no & yes & Lipoleiomyoma & no & $\begin{array}{l}\text { Chakravarty-Vartak et al. BHJ Epub } \\
\text { April } 2007\end{array}$ \\
\hline 1 & $18 \mathrm{~cm}$ & 80 & Asymptomatic & $\mathrm{TA}$ & yes & yes & yes & Lipoleiomyoma & no & $\begin{array}{l}\text { Fujiwaki et al. Arch } \\
\text { GynecolObstet:277;471-474, } 2008\end{array}$ \\
\hline 1 & $55 \mathrm{~cm}$ & 43 & Constipation & $\mathrm{TA}$ & no & no & yes & Lipoleiomyoma & no & $\begin{array}{l}\text { Akhulut et al. Arch Gynecol } \\
\text { Obstet:278;291-295, } 2008\end{array}$ \\
\hline 1 & $2 \mathrm{~cm}$ & 66 & Asymptomatic & TVS & yes & no & no & $\mathrm{UFT}^{\circ}$ & no & $\begin{array}{l}\text { Mylona et al. Eur Clinics } \\
\text { ObsteGynaecol: 3;135-136,2008 }\end{array}$ \\
\hline 1 & $8 \mathrm{~cm}$ & 68 & Asymptomatic & $\mathrm{TA}$ & yes & yes & yes & Lipoleiomyoma & no & $\begin{array}{l}\text { Loffroy et al. GynecolObstetInvest: } \\
66 ; 73-75 ; 2008\end{array}$ \\
\hline 1 & $5 \mathrm{~cm}$ & 48 & Abdominal pain & $\mathrm{TA}$ & no & no & yes & $\begin{array}{l}\text { Lipoma + ovarian } \\
\text { thecoma }\end{array}$ & no & $\begin{array}{l}\text { Vilallonga et al. Hindawi Publish Corp } \\
\text { ID 340603, } 2009\end{array}$ \\
\hline 1 & & 58 & $\begin{array}{l}\text { Abdominal pain and } \\
\text { Bleeding }\end{array}$ & & & & yes & Lipoleiomyoma & & $\begin{array}{l}\text { Mignogna et al. Arch Gynecol } \\
\text { Obstet:280;1071-74, } 2009\end{array}$ \\
\hline 1 & $3.5 \mathrm{~cm}$ & 50 & Bleeding & TSV & no & no & yes & Lipoleiomyoma & yes & $\begin{array}{l}\text { Manjunatha et al. J Midlife } \\
\text { Heath:1;86-88, } 2010\end{array}$ \\
\hline 1 & $6 \mathrm{~cm}$ & 70 & Abdominal pain & $\mathrm{TA}$ & no & no & yes & Lipoma (calcified) & no & $\begin{array}{l}\text { Bandopadhyay et al. J Turkish- } \\
\text { German Gynecol Assoc:11;113-4, } \\
2010\end{array}$ \\
\hline $\begin{array}{l}1 \\
1\end{array}$ & $\begin{array}{l}14 \mathrm{~cm} \\
6 \mathrm{~cm}\end{array}$ & $\begin{array}{l}61 \\
63\end{array}$ & $\begin{array}{l}\text { Pelvic mass } \\
\text { Abdominal pain Bleeding }\end{array}$ & $\begin{array}{l}\text { TA } \\
\text { TSV }\end{array}$ & $\begin{array}{l}\text { yes } \\
\text { no }\end{array}$ & $\begin{array}{l}\text { no } \\
\text { no }\end{array}$ & $\begin{array}{l}\text { yes } \\
\text { yes }\end{array}$ & $\begin{array}{l}\text { Lipoleiomyoma } \\
\text { Lipoma }\end{array}$ & $\begin{array}{l}\text { no } \\
\text { no }\end{array}$ & $\begin{array}{l}\text { Bindra, Sharma. The Internet J of } \\
\text { Gynecol Obstet: 12, } 2010 \\
\text { Vamseedhar et al. India J Cancer:48; } \\
385-387,2011\end{array}$ \\
\hline 1 & $9 \mathrm{~cm}$ & 70 & Bleeding and pelvic mass & $\mathrm{TA}$ & CT & no & yes & Lipoleiomyoma & no & $\begin{array}{l}\text { Terada T. Ann Diagn Pathol: Epub } \\
\text { June 6;2011 }\end{array}$ \\
\hline 3 & $\begin{array}{l}2.4 \mathrm{~cm} \\
12 \mathrm{~cm} \\
2.4 \mathrm{~cm}\end{array}$ & $\begin{array}{l}79 \\
61 \\
72\end{array}$ & $\begin{array}{l}\text { Cholecistic pain } \\
\text { Abdominal pain- } \\
\text { cholecistic pain } \\
\text { Intermittent abdominal } \\
\text { pain }\end{array}$ & $\begin{array}{l}\text { TA } \\
\text { TA } \\
\text { TSV }\end{array}$ & $\begin{array}{l}\text { yes } \\
\text { yes } \\
\text { yes }\end{array}$ & $\begin{array}{l}\text { yes } \\
\text { yes } \\
\text { yes }\end{array}$ & $\begin{array}{l}\text { no } \\
\text { no } \\
\text { no }\end{array}$ & $\begin{array}{l}\mathrm{UFT}^{\circ} \\
\mathrm{UFT}^{\circ} \\
\mathrm{UFT}^{\circ}\end{array}$ & $\begin{array}{l}\text { no } \\
\text { no } \\
\text { no }\end{array}$ & Chu et al. World J Radiol:4;58-62,2012 \\
\hline 1 & $3 \mathrm{~cm}$ & & Asymptomatic & TSV & yes & yes & no & $\mathrm{UFT}^{\circ}$ & no & Antony J. hcp.obgyn.net 2012 \\
\hline 2 & $\begin{array}{l}9 \mathrm{~cm} \\
1.5 \mathrm{~cm}\end{array}$ & $\begin{array}{l}58 \\
60\end{array}$ & $\begin{array}{l}\text { Dysuria } \\
\text { Uterine prolapse }\end{array}$ & $\begin{array}{l}\text { no } \\
\text { no }\end{array}$ & $\begin{array}{l}\text { no } \\
\text { no }\end{array}$ & $\begin{array}{l}\text { no } \\
\text { no }\end{array}$ & $\begin{array}{l}\text { yes } \\
\text { yes }\end{array}$ & $\begin{array}{l}\text { Lipoleiomyoma } \\
\text { Lipoleiomyoma }\end{array}$ & $\begin{array}{l}\text { no } \\
\text { no }\end{array}$ & Singh et al. JCDR:6;718-719, 2012 \\
\hline 1 & $2 \mathrm{~cm}$ & 37 & Pelvic pain & TSV & yes & yes & no & UFT $^{\circ}$ & no & $\begin{array}{l}\text { Loberant et al. Imaging Science Today } \\
2012\end{array}$ \\
\hline $70^{\wedge}$ & $7.7^{*}$ & $57^{\star}$ & & & & & & & & \\
\hline
\end{tabular}

Table 1: Clinical case reports. 
Citation: Bernardini L, Zacutti A, Gorji N, Giannoni ML, Accorsi F, et al. (2014) A Case Report and Review of the Literature on Uterine Fatty Tumours (UFT): A Field of Heterogeneous Data. Int J Gynecol Clin Pract 1: 101. doi: http://dx.doi.org/10.15344/2394-4986/2014/101

Page 5 of 7

\begin{tabular}{|c|c|c|c|c|c|c|c|c|}
\hline $\begin{array}{l}\text { No. of } \\
\text { cases }\end{array}$ & Age & Size & Symptoms & Histology & Fibroids & Incidence & Study period & References \\
\hline $\begin{array}{l}4+21 \\
\text { cases } \\
\text { literature }\end{array}$ & $\begin{array}{l}56 \\
47 \\
64 \\
61 \\
62^{*}\end{array}$ & $\begin{array}{l}8 \mathrm{~cm} \\
8 \mathrm{~cm} \\
18 \mathrm{~cm} \\
7 \mathrm{~cm} \\
7 \mathrm{~cm}^{*}\end{array}$ & $\begin{array}{l}\text { Bleeding } \\
\text { Abdominal Pain } \\
\text { Asymptomatic } \\
\text { Bleeding }\end{array}$ & $\begin{array}{l}\text { Lipoleiomyoma } \\
\text { Lipoleiomyoma } \\
\text { Lipoma } \\
\text { Lipoleiomyoma }\end{array}$ & $\begin{array}{l}\text { no } \\
\text { no } \\
\text { yes } \\
\text { no }\end{array}$ & & 1965-1976 & $\begin{array}{l}\text { Willen et al. Virch Arch A Path } \\
\text { Anat Histol:377;351-361, } 1978\end{array}$ \\
\hline 3 & $\begin{array}{l}83 \\
80 \\
73\end{array}$ & $\begin{array}{l}20 \mathrm{~cm} \\
2.5 \mathrm{~cm} \\
2 \mathrm{~cm}\end{array}$ & $\begin{array}{l}\text { Enlarged abdomen } \\
\text { Asymptomatic } \\
\text { Bleeding }\end{array}$ & $\begin{array}{l}\text { Lipoleiomyoma } \\
\text { Lipoleiomyoma } \\
\text { Lipoleiomyoma }\end{array}$ & $\begin{array}{l}\text { yes } \\
\text { yes } \\
\text { no }\end{array}$ & $3 / 54000(0.005 \%)$ & 1979-1982 & $\begin{array}{l}\text { Pounder. J Clin } \\
\text { Pathol:35;1380-1383, } 1982\end{array}$ \\
\hline 1 & & & $\begin{array}{l}\text { Translocation } \\
\text { Chromos } 12 \\
\end{array}$ & Lipoleiomyoma & yes & & & $\begin{array}{l}\text { Havel et al. Virch Arch B Cell } \\
\text { Path:57;77-79, } 1989\end{array}$ \\
\hline 1 & 55 & & Cholecystic pain & Atypical lipoleiomyoma & & & & $\begin{array}{l}\text { Lin and Hanai. Pathol } \\
\text { International: 41; 164-169. } 1991\end{array}$ \\
\hline 1 & & & & $\begin{array}{l}\text { Bizarre epitheliod } \\
\text { Lipoleiomyoma }\end{array}$ & & & & $\begin{array}{l}\text { Brooks et al. Int J Gynecol } \\
\text { Pathol:11;144-9, } 1992\end{array}$ \\
\hline 10 & & & & $\begin{array}{l}9 \text { lipoleiomyomas } \\
1 \text { lipoma }\end{array}$ & & & & $\begin{array}{l}\text { Resta et al. Pathol Res } \\
\text { Pract:190;378-83, } 1994\end{array}$ \\
\hline 5 & $\begin{array}{l}78 \\
67 \\
73 \\
74 \\
60\end{array}$ & $\begin{array}{l}5.3 \mathrm{~cm} \\
7 \mathrm{~cm} \\
3.5 \mathrm{~cm} \\
3.6 \mathrm{~cm}\end{array}$ & $\begin{array}{l}1 \text { angiomyolipoma } \\
4 \text { lipoleiomyoma }\end{array}$ & $2 / 5$ & & & & $\begin{array}{l}\text { Shintaku. Pathol Int:46; 498-502, } \\
1996\end{array}$ \\
\hline 1 & & & & Lipoleiomyoma & & & & \\
\hline 1 & & & & Lipoleiomyoma & & $0.8 \%$ & & $\begin{array}{l}\text { Gentile et al. } \\
\text { Pathologica:88;132-4, } 1996\end{array}$ \\
\hline 1 & & & & Lipoleiomyoma & & $\begin{array}{l}0.28 \% \text { (fibroids) } \\
0.39 \% \text { (hysterectomies) }\end{array}$ & & $\begin{array}{l}\text { Dellachà et al. } \\
\text { Pathologica:89;737-41, } 1997\end{array}$ \\
\hline 17 & $45-74$ & & & $6 / 17$ angiomyolipomas & $10 / 17$ & $0.35 \%$ & $1983-2003$ & $\begin{array}{l}\text { Lin et al. Int J Gynaecol } \\
\text { Obstet:67;47-9, } 1999\end{array}$ \\
\hline 1 & 57 & $11 \mathrm{~cm}$ & Bleeding & Plexiform lipoleiomyoma & & & & $\begin{array}{l}\text { Morelli et al. Arch Gynecol } \\
\text { Obstet:274;117-118, } 2006\end{array}$ \\
\hline 50 & $54^{*}$ & $4.6^{*} \mathrm{~cm}$ & & Lipoleiomyoma & & $2.1 \%$ & 1998-2004 & $\begin{array}{l}\text { Wang et al. Int J Gynecol } \\
\text { Pathol:25;239-42, } 2006\end{array}$ \\
\hline 3 & & & & Lipoleiomyoma & & & & $\begin{array}{l}\text { Kondi-Pafiti et al. Eur j Gynaecol } \\
\text { Oncol:27;73-7, } 2006\end{array}$ \\
\hline 10 & $53^{*}$ & $4.75^{\star} \mathrm{cm}$ & Pain, Bleeding & Lipoleiomyoma & & $1.4 \%$ & 1999-2007 & $\begin{array}{l}\text { Bolat et al. Turkish J Pathol: 23: } \\
82-86,2007\end{array}$ \\
\hline 2 & $\begin{array}{l}47 \\
66\end{array}$ & $\begin{array}{l}9 \mathrm{~cm} \\
30 \mathrm{~cm}\end{array}$ & & $\begin{array}{l}\text { Lipoleiomyoma } \\
\text { Giant lipoleiomyoma }\end{array}$ & $\begin{array}{l}\text { no } \\
\text { no }\end{array}$ & & & $\begin{array}{l}\text { Terada T. Appl } \\
\text { Immunohistochem Mol Morphol: } \\
\text { Epub Jan 26, } 2012\end{array}$ \\
\hline $132^{\wedge}$ & $65^{*}$ & $9.6^{*}$ & & & & & & \\
\hline
\end{tabular}

Table 2: Pathology review studies.

$\wedge$ Total $\mathrm{n}$ of cases ${ }^{\star}$ mean values 
Citation: Bernardini L, Zacutti A, Gorji N, Giannoni ML, Accorsi F, et al. (2014) A Case Report and Review of the Literature on Uterine Fatty Tumours (UFT): A Field of Heterogeneous Data. Int J Gynecol Clin Pract 1: 101. doi: http://dx.doi.org/10.15344/2394-4986/2014/101

Page 6 of 7

\begin{tabular}{|c|c|c|}
\hline $\begin{array}{l}\text { No. of } \\
\text { cases }\end{array}$ & Histology & Reference \\
\hline 1 & $\begin{array}{l}\text { Uterine lipoma and } \\
\text { endometrial cancer }\end{array}$ & $\begin{array}{l}\text { Tlolka Pluszczyk et al.Patologia } \\
\text { Polska:36;223-227, } 1968\end{array}$ \\
\hline 1 & $\begin{array}{l}\text { Uterine sarcoma with } \\
\text { liposarcomatous diff. }\end{array}$ & $\begin{array}{l}\text { Bapat et al. Int J Gynaecol } \\
\text { Obstet:28;71-5, } 1989\end{array}$ \\
\hline 1 & $\begin{array}{l}\text { Uterine lipoma and } \\
\text { endometrial cancer }\end{array}$ & $\begin{array}{l}\text { Douvier et al. J Gynecol Obstet Biol } \\
\text { Reprod:19;301-305, } 1990\end{array}$ \\
\hline 2 & $\begin{array}{l}\text { Sarcomas with both lipo } \\
\text { and leiomyo-sarcoma cells }\end{array}$ & $\begin{array}{l}\text { Suster et al. Am J Surg } \\
\text { Pathol:17;905-911, } 1993\end{array}$ \\
\hline 1 & Liposarcoma of the uterus & $\begin{array}{l}\text { Schneebauer et al. GynakolGeburt } \\
\text { Rundsch:36;90-91, } 1996\end{array}$ \\
\hline 1 & $\begin{array}{l}\text { Uterine lipoma and } \\
\text { endometrial cancer }\end{array}$ & $\begin{array}{l}\text { Di Gesù et al.Eur J Obstet Gynecol } \\
\text { Reprod Biol:80;199-200, } 1998\end{array}$ \\
\hline 9 & Lipoleiomyosarcoma & $\begin{array}{l}\text { Folpe, Weiss. Am J Surg } \\
\text { Pathol:26;742-9, } 2002\end{array}$ \\
\hline 1 & Pleomorphic liposarcoma & $\begin{array}{l}\text { Levine et al. Int J Gynecol } \\
\text { Pathol:22;407-411, } 2003\end{array}$ \\
\hline 1 & Liposarcoma & $\begin{array}{l}\text { Karateke et al. Int J Gynecol } \\
\text { Cancer:15;1230-1234, } 2005\end{array}$ \\
\hline 1 & $\begin{array}{l}\text { Uterine lipoma and cervical } \\
\text { cancer }\end{array}$ & $\begin{array}{l}\text { Dilek et al. Int J Gynecol } \\
\text { Cancer:16;445-7, } 2006\end{array}$ \\
\hline 1 & Lipoleiomyosarcoma & $\begin{array}{l}\text { AbhimanyuJha et al. NJ Obstet } \\
\text { Gynaecol:2;67-70, } 2007\end{array}$ \\
\hline 1 & $\begin{array}{l}\text { Uterine lipoleiomyoma and } \\
\text { endometrial cancer }\end{array}$ & $\begin{array}{l}\text { Bolat et al. Turkish J Pathol:2382-86, } \\
2007\end{array}$ \\
\hline 3 & $\begin{array}{l}\text { Liposarcoma arising in } \\
\text { uterine lipoleiomyoma }\end{array}$ & $\begin{array}{l}\text { McDonald et al. Am J Surg } \\
\text { Pathos:35;221-227, } 2011\end{array}$ \\
\hline
\end{tabular}

Table 3: UFT: Oncological data (association with uterine cancers in $24 / 226$ cases $=10 \%$ ).

to study their incidence $[23,24]$. Despite all the UFT appear similar (with a bright yellow colour and soft tissue consistency) they show, after microscopy, different histological constitution. The high range of histopathological appearance has caused a proliferation of synonyms for UFT. The more common synonyms include lipoleiomyoma, myolipoma, lipofibroma, lipomyoma, fibromyolipoma, mixed lipoma, and lipomatosis of the stroma of a uterine fibroid. According to DJ Pounder (1982) [25] uterine fatty tumours (UFT) may be defined as tumours composed entirely or in part of adult type adipose tissue. Smooth muscle and fibrous tissue are usually intermixed. The presence of fat in the uterine corpus is not exceptional and in fact it is known that some leiomyomas have an adipose tissue component in variable proportions associated to smooth muscular fibres. These are known as lipoleiomyomas and have to be considered aside from the pure lipomas which exclusively comprise of mature adipose tissue. The pathogenesis however remains obscure. Immunocytochemical studies confirm the complex histogenesis of these tumours, which may directly arise from pluripotent mesenchymal cells or from direct transformation of smooth muscle cells into adipocytes [26,27]. Probably we are dealing with tumour-types having different pathogenesis and therefore different biological susceptibility to oncogenes. A number of various lipid metabolic disorders or other associated conditions with estrogen deficiency as occur in peri or post menopausal period possibly promote abnormal intracellular storage of lipids [28]. As shown by Terada [24] the fatty tissue of lipoma is not degenerative but active proliferative tissue and could be responsible of local productions of estrogens and increased risk of malignant transformation. Wang et al [3] studying 50 patients with UFT for a 7 years period of time have reported that these tumours have an uneventful clinical course and should be confidently regarded as benign. Nevertheless, liposarcomas of the uterus, although extremely rare, exist and are shown to likely arise from malignant transformation of a lipoleiomyoma [29] and have to be added to the differential diagnosis of benign lipomatous tumours (UFT), myxoid mesenchymal tumours, and malignant mixed Mullerian tumours of the uterus. The striking observation of endometrial cancers found in concomitance with lipomas, as here reported, has an independent and different oncological implication. Deeper investigation on this field is requested before making progress on the oncological risk of the UFT which remain rare tumours undergoing hysterectomy most of the times.

\section{Conclusion}

UFT are not so uncommon and always benign neoplasm as generally stated. The histogenesis of these lesions is still controversial. The clinical manifestations do not usually differ from those caused by leiomyomas, except that they affect overweight and obese perimenopausal and aged postmenopausal women. Data on body weight and BMI, however, are missing and should better be given in future. Preoperative diagnosis it is not difficult as far as these tumours are of small size and appear hyperechogenic intramural nodules at transvaginal ultrasound. A more difficult task is in case of big and subserosal tumours whose accurate diagnosis requires CT and MR imaging. Diagnosis of pure lipoma rather lipoleiomyoma should be made only postoperatively on histopathology which is also important to rule out the possibility of malignancy. The adoption of proper terminology ("uterine fatty tumours - UFT)" definition in clinical studies and "lipoma" or "lipoleiomyoma" after histology, should be respected.

\begin{tabular}{|c|c|c|c|c|c|c|c|}
\hline $\begin{array}{l}\text { Total number of } \\
\text { cases ( } 357 \text { cases) } \\
1816-1966=131\end{array}$ & Mean age (years) & $\begin{array}{l}\text { Prevalence } \\
\text { in women }< \\
54 \text { y.o. }\end{array}$ & Mean size $(\mathrm{cm})$ & $\begin{array}{l}\text { Pre-op radiol } \\
\text { studies (46) }\end{array}$ & $\begin{array}{l}\text { Incidence of } \\
\text { lipoma }\end{array}$ & Symptoms & Leiomyomas \\
\hline $1966-2012=226$ & $\begin{array}{l}\text { Table } 1(47 \text { cases })=57 \\
\text { Table } 2(16 \text { cases })=65 \\
\text { Willen st. }(21 \text { cases })=62 \\
\text { Wang study }(50 \text { cases })=54 \\
\text { Bolat study }(10 \text { cases })=53\end{array}$ & $23 \%(19 / 83)$ & $\begin{array}{l}\text { Table } 1(46 \text { cases })=7.7 \\
\text { Table } 2(14 \text { cases })=9.6 \\
\text { Willen study }(21 \text { cases })=7 \\
\text { Wang study }(50 \text { cases })=4.6 \\
\text { Bolat study }(10 \text { cases })=4.75\end{array}$ & $\begin{array}{l}16 / 46 \text { (us }+\mathrm{ct}+\mathrm{mr} \text { ) } \\
4 / 46 \mathrm{tsv} \text { us only } \\
2 / 46 \mathrm{mr} \text { only }\end{array}$ & $\begin{array}{l}15 \%(18 / 121 \\
\text { cases) }\end{array}$ & $\begin{array}{l}34 \%(17 / 50) \\
\text { pain or pelvic } \\
\text { mass } \\
22 \%(11 / 50) \\
\text { no symptoms } \\
20 \%(10 / 50) \\
\text { bleeding } \\
10 \%(5 / 50) \\
\text { cholecistic } \\
\text { problems }\end{array}$ & $33.3 \%(34 / 102)$ \\
\hline
\end{tabular}

Table 4: Summary of principal variables on UFT. 
Citation: Bernardini L, Zacutti A, Gorji N, Giannoni ML, Accorsi F, et al. (2014) A Case Report and Review of the Literature on Uterine Fatty Tumours (UFT): A Field of Heterogeneous Data. Int J Gynecol Clin Pract 1: 101. doi: http://dx.doi.org/10.15344/2394-4986/2014/101

\section{References}

1. Jacobs JE, Markowitz SK (1988) CT diagnosis of uterine lipoma. AJR Am J Roentgenol 150: 1335-1336.

2. Willén R, Gad A, Willén H (1978) Lipomatous lesions of the uterus. Virchows Arch A Pathol Anat Histol 377: 351-361.

3. Wang X, Kumar D, Seidman JD (2006) Uterine lipoleiomyomas: clinicopathologic study of 50 cases. Int J Gynecol Pathol 25: 239-242.

4. Vilallonga R, Garcia A, Castellvi J, Fort JM, Armengol M, et al. (2009) Lipoma of the Uterine Corpus: Exceptional Eventuality Combined with an Ovarian Thecoma. Case Reports in Medicine 2009: 340603.

5. Bolat F, Kayaselcuk F, Canpolat T, Serkan E, İlhan T (2007) Histogenesis of lipomatous component in uterine lipoleiomyomas. Turkish $\mathrm{J}$ of Pathology 23: $82-86$

6. Dodd GD 3rd, Budzik RF Jr (1990) Lipomatous tumors of the pelvis in women: spectrum of imaging findings. AJR Am J Roentgenol 155: 317-322.

7. Chan HHL, Chau MT, Lam CHL, Cheung SCW (2003) Uterine lipoleiomyoma: ultrasound and computed tomography findings. JHK Coll Radiol 6: 30-32.

8. Prieto A, Crespo C, Pardo A, Docal I, Calzada J (2000) Uterine lipoleiomyomas: US and CT findings. Abdom Imaging 25: 655-657.

9. Chu CY, Tang YK, Chan TS, Wan YH, Fung KH (2012) Diagnostic challenge of lipomatous uterine tumors in three patients. World J Radiol 4: 58-62.

10. Kitajima K1, Kaji Y, Imanaka K, Sugihara R, Sugimura K (2007) MR findings of uterine lipoleiomyoma correlated with pathologic findings. AJR Am J Roentgenol 189: W100-W104

11. Jha A, Sayami G, Adhikari D (2007) Lipoleiomyosarcoma an extremely unusual sarcoma of uterus: a case report. NJ Obstet Gynaecol 2: 67-70.

12. Chakravarty U S, Dhurde A V, Vartak SS, Badarkhe G, Veer S (2008) Vaginal Angiomyofibroblastoma. Bombay Hospital Journal 50: 1.

13. Antony J. Lipoleiomyoma of the uterus. www.hcp.obgyn.net/blog/ultrasound

14. Loberant N, Bhatt S, Messing E, Dogra SV (2011) Bilateral Testicular Epidermoid Cysts J Clin Imaging Sci 1: 4.

15. Keeling AN, Reidy JF (2007) Imaging and treatment of uterine fibroids, including the role of uterine artery embolization. Imaging 19: 374-384.

16. Pham CA, Atri M, Senterman MK (1993) Ultrasonographic appearance of uterine lipoleiomyoma. Can Assoc Radiol J 44: 463-465.

17. Serafini G, Martinoli C, Quadri P, Speca S, Crespi G, et al. (1996) Lipomatous tumors of the uterus: ultrasonographic findings in 11 cases. $J$ Ultrasound Med 15: 195-199.

18. Braun HL, Wheelock JB, Amaker BH, Seeds JW (2002) Sonographic evaluation of a uterine angiolipoleiomyoma. J Clin Ultrasound 30: 241-244.

19. Avritscher R, lyer RB, Ro J, Whitman G (2001) Lipoleiomyoma of the uterus. AJR Am J Roentgenol 177: 856.

20. Fujimoto Y, Kasai K, Furuya M, Honda N, Tojo R, et al. (2006) Pure uterine lipoma. J Obstet Gynaecol Res 32: 520-523.

21. Manjunatha HK, Ramaswamy AS, Kumar BS, Kumar SP, Krishna L (2010) Lipoleiomyoma of uterus in a postmenopausal woman. J Midlife Health 1 : 86-88

22. Fujiwaki $\mathrm{R}$, Ohnuma $\mathrm{H}$, Miura $\mathrm{H}$, Sawada $\mathrm{K}$ (2008) Uterine lipoleiomyoma in an elderly patient: a case report. Arch Gynecol Obstet 277: 471-474.

23. Lau LU, Thoeni RF (2005) Case report. Uterine lipoma: advantage of MRI over ultrasound. Br J Radiol 78: 72-74.
24. Terada T (2011) Large lipoleiomyoma of the uterine body. Ann Diagn Pathol 16: 302-305.

25. Pounder DJ (1982) Fatty tumours of the uterus. J Clin Pathol 35: 13801383.

26. Shintaku M (1996) Lipoleiomyomatous tumors of the uterus: a heterogeneous group? Histopathological study of five cases. Pathol Int 46 498-502

27. Resta L, Maiorano E, Piscitelli D, Botticella MA (1994) Lipomatous tumors of the uterus. Clinico-pathological features of 10 cases with immunocytochemical study of histogenesis. Pathol Res Pract 190: 378383.

28. Lin KC, Sheu BC, Huang SC (1999) Lipoleiomyoma of the uterus. Int J Gynaecol Obstet 67: 47-49.

29. McDonald AG, Dal Cin P, Ganguly A, Campbell S, Imai Y, et al. (2011) Liposarcoma arising in uterine lipoleiomyoma: a report of 3 cases and review of the literature. Am J Surg Pathol 35: 221-227. 